TITLE:

\title{
Reconsideration of animal models of schizophrenia and other psychiatric disorders with evolutionary perspective.
}

$\operatorname{AUTHOR}(S):$

Lee, Young-A; Goto, Yukiori

\section{CITATION:}

Lee, Young-A ... [et al]. Reconsideration of animal models of schizophrenia and other psychiatric disorders with evolutionary perspective.. Medical hypotheses 2013, 81(6): 1120-1126

\section{ISSUE DATE:}

2013-12

URL:

http://hdl.handle.net/2433/179780

\section{RIGHT:}

(C) 2013 Elsevier Ltd.; この論文は著者最終稿です。内容が印刷版と異な ることがありますので、引用の際には出版社版をご確認ご利用くださ $\omega_{\circ}$ This is the Accepted Author Manuscript. Please cite only the published version. 


\title{
Reconsideration of Animal Models of Schizophrenia and Other Psychiatric Disorders with Evolutionary Perspective
}

\author{
Young-A Lee, Ph.D. \& Yukiori Goto, Ph.D.* \\ Kyoto University Primate Research Institute, Aichi, Japan
}

\section{Correspondence:}

Yukiori Goto, Ph.D.

Kyoto University Primate Research Institute

41-2 Kanrin, Inuyama,

Aichi 484-8506

Japan

Phone: +81(568)63-0551

Fax: +81(598)63-0551

E-mail: goto.yukiori.5c@kyoto-u.ac.jp

Number of Pages: 29 pages

Title: 13 words

Abstract: 97 words

Text: 4,033 words

References: 72

Figures: 5

Tables: 0

\section{Acknowledgements}

The authors are supported by Kyoto University. We thank Dr. Nobuo Masataka for support. 


\section{Abstract}

Studies utilizing animal models for understanding biological mechanisms of such psychiatric disorders as schizophrenia have been now flourishing. Animal models are a essential part of translational research, and without them, it is not possible to develop therapeutic strategies to treat psychiatric disorders. Accordingly, importance of animal models have been increasingly emphasized. However, on the other side, limitations of such an animal model approach has been growingly deceptive. The aim of this review article is to discuss limitations of translational research utilizing animal models, and propose a new direction of research with evolutionary perspective to understand psychiatric disorders.

Keywords: Animal model, Schizophrenia, Evolution, Translational research, Environmental adaptation, Psychiatric disorder 


\section{Introduction}

Schizophrenia is a psychiatric disorder that has been under extensive investigation for long time. Schizophrenia had been once said "the graveyard of neuropathologists" (1) because of unclear pathological changes in the brains of schizophrenia patients. However, tremendous efforts, especially with recent progresses of live human imaging techniques as well as genetic understanding and engineering technologies have made significant advancement for unveiling the mystery of schizophrenia. Consequently, an optimistic belief has emerged that biological understanding of this psychiatric disorder is now within the hand, and we will eventually be able to cure the disorder. Nevertheless, the fact is that, for treatments of schizophrenia and most of other psychiatric disorders, we still do not have therapeutic methods based on the fruits of recent research, and have to rely on drugs that are based on the old serendipitous findings with considerable devastating side effects and that works in some, but not all patients.

Translational research, which is the process that develops new and better therapeutic drugs and strategies for human psychiatric disorders by applying findings in basic scientific research utilizing animals, has been currently a main research strategy (Fig. 1) (2, 3). Animal models consist of an essential part of the translational research. Accordingly, importance of animal models have been increasingly emphasized. In contrast, limitations of utilizing animal models to understand the biological mechanisms of psychiatric disorders has tended to be disregarded. This review articles discusses limitations of translational research utilizing animal models for understanding such psychiatric disorders as schizophrenia, and provides a novel, alternative approach to 
understand psychiatric disorders with evolutionary perspective.

\section{Animal models of schizophrenia}

Following three criteria have been proposed that animal models of psychiatric disorders have to fulfill; [i] construct validity, which is whether manipulations given in animals to create models have relations to the suggested causes of the disorders; [ii] face validity, which is whether alterations observed in animal models resemble to the symptoms of the disorders; and (4) predictive (pharmacological) validity, which is whether alterations in animal models are ameliorated by currently used pharmacotherapeutic drugs or other treatment methods in human patients (5).

Animal models of schizophrenia particularly require the following additional criteria (6); augmented responses to such psychostimulants as amphetamine and phencyclidine (PCP) and stress, which have been shown to precipitate or exacerbate symptoms in schizophrenia patients; decreased social interaction with mates, which resembles to the negative symptoms of schizophrenia; deficits in such sensory gating mechanisms as prepulse inhibition; and cognitive dysfunction, especially those relating to prefrontal cortical (PFC) function such as working memory, attention, and behavioral flexibility. However, the criterion that appears to be most critical and relevant to schizophrenia is delayed onset of abnormalities. Thus, models involve manipulations given in early development (i.e. prenatal and neonatal periods), but abnormalities are quiescent in childhood, but become apparent when animals reach young adulthood. 
This development-dependent emergence of abnormalities resembles the typical onset age of the schizophrenia symptoms in late adolescence to young adulthood, especially in male patients (female patients also have the peak of onset age in early adulthood, but they have another second peak on later ages around 40 to 70 years old (7). Such male-female difference of the onset age of the symptoms have been barely considered in animal model studies).

To date, many animal models of schizophrenia have been proposed, which can be roughly divided into (1) lesion models; (2) pharmacological models; and (3) genetic manipulation models. Lesion models include neonatal hippocampus (8) and PFC lesion (9) in rodents as well as neonatal temporal lobe lesion in non-human primates (10). These lesion models emphasize the face validity, which exhibit an assortment of behavioral alterations that are similar to the symptoms and abnormalities observed in schizophrenia patients. However, these models are clearly short of the construct validity, as no clear brain damage has been confirmed in schizophrenia brains. Pharmacological models include prenatal exposure to methylazoxymethanol acetate (11), prenatal immune activation (12), and exposure to such psychostimulants as amphetamine (13) and PCP (14). In pharmacological models, both construct and face validities are balanced. Currently the main stream of animal model research is utilization of genetic models. As more schizophrenia candidate genes are recently identified, an expanding number of genetic manipulation models have been now proposed $(15,16)$. Genetic models emphasize the construct validity, but the models do not necessarily have to have a good face validity, as the aim of these genetic models is on endophenotype analyses, correlating dysfunction of a specific gene and a specific aspect of an abnormality observed in the disorder. 


\section{Limitations of animal model approach}

Translational research bases on the idea that fundamental molecular, biochemical, anatomical, and physiological architectures of animals (including rodents and non-human primates) and human brains are similar, such that findings in animals can be readily applicable to humans. This may be partly true, but it is more likely a naive presumption. However, limitations of translational research with utilization of animal models have been barely discussed to date.

One example that coins limitations of translational research with animal model approach is the case of the metabotropic glutamate receptor (mGluR) II/III agonist developed by the pharmaceutical company Eli Lilly. The study by Moghaddam and Adams published in Science in 1998 (17) is the first that suggests potential therapeutic effects of an mGluRII/III agonist on schizophrenia symptoms. They reported that the mGluRII/III agonist could reverse PCP-induced alterations in rodents. In synergy with the glutamatergic hypothesis of schizophrenia (18) and subsequent many animal model studies supporting this first finding $(19,20)$, it appeared that the mGluRII/III agonist was the most promising novel therapeutic drug against schizophrenia. Eli Lilly then developed the mGluRII/III agonist LY2140023, and conducted clinical trials. The first phase II trial revealed significant therapeutic effects (21), but twice of subsequent phase II trials resulted in observation of no therapeutic effects of this drug. The reason why the drug had therapeutic effects in the first phase II trial, but not in subsequent trials, is 
unknown. However, the most important point is that in animal model studies, almost all cases have clearly demonstrated significant therapeutic potential of the mGluRII/III agonist.

Although this failure may be associated with difference of drug metabolisms that determine pharmacological responses between humans and animals, it is possible that it may also reflect limitations of animal model approach.

First, the limitation is associated with a question whether human brain is a scaled-up version of that in such animals as non-human primates and rodents. For instance, Elston and colleagues have shown that dendritic spine density of cortical neurons in humans are about twice of that in rhesus macaques and triple of that in marmosets (22). This finding can be interpreted in two ways. One is that human brain is scaled-up version of monkey brain. The current translational research is based on this interpretation. Indeed, brains of humans and animals including non-human primates undoubtedly share some common mechanisms. The other is that this difference of the spine density is a crucial factor that distinguishes humans and non-human primates and something that we should not ignore. If emergence of such psychiatric disorders as schizophrenia originates in the common mechanisms between humans and animals, the current translational research will eventually come up into understanding of the disorders and development of new therapeutic strategies. On the other hand, if emergence of the disorders may nest in such difference between humans and animals, the current approach of translational research may not be able to reach the answer.

The second limitation is that the criteria for animal models are mostly based on indecisive evidence of human cases. In schizophrenia, a number of promising 
molecular, anatomical, and physiological changes have been reported (23). However, most of them have not been well replicated. Nevertheless, these alterations, although supported by only weak evidence, have been accepted as the criteria in animal models. For instance, cognitive dysfunction has been suggested as the "core" deficit underlying schizophrenia (24). Cognitive dysfunction can be also assessed in animals, such that cognitive dysfunction has been utilized as an important criterion in animal models of schizophrenia. Nonetheless, it is particularly important to note that not all schizophrenia patients exhibit cognitive deficits. In fact, it has been estimated that up to $75 \%$ of schizophrenia patients exhibit clear cognitive dysfunction, suggesting that cognitive function in at least one fourth of schizophrenia patients are still within a normal level (25). One explanation for this observation is that some schizophrenic patients have even higher cognitive function than normal subjects before symptom onset, and cognitive decline results in normal range of function in these patients. However, this argument does not explain why such schizophrenic patients with normal level of cognitive function still have to suffer from the symptoms and social malfunctioning. A feasibility for the argument of cognitive dysfunction as the core deficit of schizophrenia is further weakened by the fact that cognitive dysfunction can be observed in most of other psychiatric disorders. A deficit in sensory gating such as prepulse inhibition has been also considered as an important factor in schizophrenia. However, deficits in prepulse inhibition are also observed in other psychiatric conditions (26-28).

The third limitation involves assessment methods for animal models. For instance, decreased amount of social interaction with mates is one of the important criteria utilized in animal models of schizophrenia (6). However, it is not possible to assess whether such decreased social interaction is due to increased fear, decreased 
interest, or decreased motivation to interact with mates. In schizophrenia, decreased social interaction may be associated with a mixture of fear and decreased motivation to interact with others, whereas in autism, it may be associated with a mixture of increased fear and decreased interest to interact with others. Although outcome appears to be very similar, if not identical, the mechanisms underlying the outcome are often different, which cannot be distinguished in animal models.

It is interesting to note that a similar difficulty on developing new and better therapeutic drugs and treatment methods for stroke based on animal models has been also raised (29). In animal model studies, almost 500 therapeutic drugs and methods have been suggested to be effective for treatments of stroke; nevertheless, only two drugs (aspirin and recombinant tissue plasminogen activator) have been confirmed to yield true effectiveness in human patients.

\section{Psychiatric disorder-associated behavior in animal society}

In addition to the above discussion, another question is whether it is really appropriate to construct psychiatric disorder models using animals themselves.

Psychiatric and neurological disorders have been barely reported in wild animals including non-human primates. There is no report of psychiatric disorders even in such hominidae primates as chimpanzees, orangutans, and gorillas that are species closest to humans. There are two possible explanations for this fact. One is that psychiatric conditions happen only with the human brain structure. Alternately, 
psychiatric conditions may present in animals, but such conditions exist as normal ones to them. Psychiatric disorders are considered as maladaptation causing disadvantages within the human social system constructed by the majority of so-called "normal" people (Fig. 2). When the social systems in which organisms are living are different, then meaning of psychiatric disorder-associated behavior becomes different. For instance, if subjects with schizophrenia became a majority, and so-called normal people became a minority in the human social system, the structure of the social system could be reorganized such that schizophrenia symptoms were accepted as normal or advantageous behavior in the reorganized social system. Therefore, psychiatric disorder-associated behavior may not necessarily be disadvantages, but whether such behavior is advantageous or disadvantageous can be determined only in relation with environments in which organisms are living. One example illustrating this aspect is attention deficit/hyperactivity disorder (ADHD) (30). ADHD consists of hyperactivity, impulsivity, and attention deficit with incapability to sustain attention to a particular target. These symptoms are maladaptive and disadvantageous in the modern human social system such as school. However, these symptoms are clearly advantageous in environment where a life-threatening danger is approaching. Hyperactivity and inattention enables to detect quicker detection, and impulsivity enables to make quick decision to escape from the danger.

Therefore, it is possible that normal behavior and thereby brain function and structures of animals may be already similar to the conditions of psychiatric disorders in humans. If this is true, a doubt arises on how useful to conduct artificial manipulations in animals whose brains are already similar to those of psychiatric conditions to create models of the disorders. 


\section{Difficulties of creating animal models of schizophrenia and autism}

A question whether animal models, especially those of schizophrenia and autism, are appropriate also comes from the oppositional relationships between these disorders. Thus, creating animal models of these disorders appears to be very difficult, based on the arguments that normal behavior and thereby normal brain function of animals may be already autistic, whereas schizophrenia may be associated with human-specific brain mechanisms.

Crespi and Badcock have suggested that schizophrenia and autism share many commonalities, but most of which are in the opposite directions, (31). Crespi and colleagues have also provided evidence in their elegant study that schizophrenia and autism are also considered oppositional disorders in genetics (32). They have shown that schizophrenia and autism share many susceptible genes. Moreover, prolongation and shortening of copy number variations in some of these genes are opposite between the disorders.

Oppositional alterations in some of brain function between schizophrenia and autism are particularly interesting. The mechanisms of visual information integration appear to be altered in both schizophrenia and autism. Gestalt perception such as Kanizsa's triangle (Fig. 3a) requires integration of visual information to see an illusionary figure (33). An embedded figures test is to find a particular shape written over complex figures (Fig. 3b), which requires dissociation of the figures into each 
component (34). Therefore, Gestalt perception and an embedded figure test require opposite visual information processing. Although results are still inconclusive, prodoromal phase or early stage of schizophrenia patients $(35,36)$ have been shown superior performance to normal subjects on gestalt perception, whereas subjects with autism $(37,38)$ are superior to normal subjects on an embedded figure test. These studies suggest that the mechanism of visual information integration is abnormally augmented in schizophrenia, whereas it is underdevelopment in autism. In relation to this issue, it has been suggested that the local neural network connections within the PFC is abnormally enhanced, whereas connections between the PFC and other brain structures are attenuated in autism (Fig. 4a) (39). Frontotemporal dementia is a neurological disorder with selective degeneration between the PFC and temporal cortex, thus producing disconnection between these two cortical regions (40). Patients with frontotemporal dementia exhibit stereotypy, restricted interest only to a specific object, impulsive and inflexible behavior, decreased spontaneous conversation, attenuated ability of visual information integration that is often expressed as an artistic ability (41), all of which are also the characteristics of autistic symptoms. In schizophrenia, dysconnectivity, with connections between the PFC and some of other brain structures abnormally augmented, has been suggested (Fig. 4b) (42). This is supported by functional imaging studies showing abnormal presence of connectivity between the PFC and temporal cortex, which is usually absent in normal subjects, in schizophrenia patients while engaging in cognitive tasks $(43,44)$.

Temple Grandin, who is autistic by herself, have suggested that behavior and the way of thinking in autism are similar to those of animals (45). There is no direct evidence to support this argument, and therefore, it is mostly speculation. However, it 
is notable that several characteristic behavior of autism such as avoidance of eye contact, which is a sign of intimation in animals, limited language communication, fear as primary emotion, and difficulty in gestalt perception, are very similar to animal-like traits. Victor of Aveyron (1788-1828) was a boy who was found living in the woods of Aveyron in the South-Central France. His behavior was described by the doctor Jean Marc Gaspard Itard. It is suggested that the behavior of Victor, if he had been living in the modern society, would have been diagnosed as autism (46). It is possible that growing in the wild environment made Victor autistic-like. Alternately, it is also possible that Victor was thrown by parents because of autism, but could survive in the wild environment owing to the autistic-traits (such as avoidance of eye contact). In relation to this issue, alterations of the theory of mind in schizophrenia and autism are also interesting. The theory of mind is an ability to estimate mental states of others. Whether the theory of mind is an unique function of humans or not is a matter of debate (47). However, even if the theory of mind is present in animals, it is likely that this function is most developed in humans compared to any other species of animals. Autism has been shown to exhibit a deficit in the theory of mind (48). A deficit in the theory of mind has been also reported in schizophrenia patients $(49,50)$. Nonetheless, the deficit in schizophrenia may be different from that in autism. With the positive symptoms such as delusion, schizophrenia patients often claim misrepresentation of mental states of others such as "neighbors are spying on them". This may be explained by an abnormally exaggerated function of the theory of mind in schizophrenia. A number of studies have shown association between a theory of mind deficit and delusion in schizophrenia patients $(51,52)$. Therefore, the schizophrenic symptom, especially psychosis, may be emerged through evolution as a disadvantageous 
by-product or advantageous change of the brain function mediating the theory of mind.

\section{Evolutionary approach for understanding psychiatric disorders}

Almost all biological psychiatric studies including translational research with animal models aim to unveil "how" psychiatric disorders are caused in humans ("proximate question" coined by Tinbergen (53)). An alternate approach is to explore "why" psychiatric disorders exist in humans (Tinbergen`s "ultimate question"). To approach this ultimate question, investigation with evolutionary perspective is critical.

The epidemiological study by Power and colleagues has reported that fecundity of psychiatric patients is significantly lower than that in normal subjects (54). In particular, fecundity of male subjects with schizophrenia and autism is only one fifth of normal subjects. Fecundity of relatives of these patients is not significantly higher than that of normal subjects. Collectively, although genetics play significant roles in both schizophrenia and autism, a chance of inheritance of susceptible genes that cause these disorders is very low. This predicts decreasing percentage of afflicted subjects among population, and disorders eventually vanish over generation. However, in reality, schizophrenia has been present among humans for as long as several thousand years, evidenced by descriptions of existence of schizophrenia-like subjects in such old scripts as Eber Papyrus (1500 BC, Egypt) and Atharva Veda (1400 BC, India) (55). The Darwinian natural selection theory predicts that disadvantageous phenotypes should have been vanished over generation. Even the theory of Lamarckian inheritance of 
acquired phenotypes predicts that disadvantageous phenotypes should not have been evolved. Therefore, evolutionary perspective suggests that psychiatric disorders may not necessarily be disadvantageous, but could be advantageous phenotypes that have evolved as environmental adaptation or (still disadvantageous) by-products of such process. Supporting evidence of this issue is still weak. However, Crespi and colleagues have shown that traces of positive selection can be found on the schizophrenia and autistic candidate genes (56). There is no direct evidence to support that schizophrenia is an advantageous change in evolution. However, there have been a number of hypothesis arguing advantageous aspects of this disorder. One of such hypotheses is the group-splitting hypothesis proposed by Stevens and Price (57). They have proposed that schizophrenia may have a beneficial role to stabilize a group by splitting it into small ones when the group becomes too large and resources to support the group is scarce. This hypothesis sounds attractive, since it is consistent with our idea discussed above that social stress yielded from over-crowdedness of the society may be driving force of evolution of the theory of mind, and psychosis may involve abnormal augmentation of the theory of mind.

Understanding biological mechanisms of psychiatric disorders as environmental adaptation with evolutionary perspective may be a fruitful direction of research that can open a new venue in the field.

\section{Investigation of psychiatric disorders with evolutionary perspective}


One way of investigation that can be an alternate over the current translational research for understanding psychiatric disorders is to examine how brain architectures and function are different between humans and animals (Fig. 5). More specifically, investigation aims to examine difference of brain structure and function between humans and animals, and elucidate why such difference is created through evolution as environmental adaptation, and how such adaptation results in emergence of psychiatric conditions in humans. In this approach, a hypothesis is that psychiatric disorders such as schizophrenia involves the brain mechanisms that are unique in humans. Such an approach is the opposite direction of the current translational research. Indeed, this approach is also different from the current research in which the current biological psychiatric research usually conduct experiments and make conclusions with only one specific species.

Another approach is use of domestic animals, especially canines, as an animal model. In particular, social function of canines is atypical and distinct from that in wild animals. Canines are highly sensitive to human social signals. For instance, Canines have been shown to be able to learn behavior by observing behavior demonstrated by humans (58). They can also use human eye signals for point-following. Such an ability is not endowed in any other animals including chimpanzees, the non-human primates suggested to be closest to humans (59). This hints a similar relationship between normal subjects and subjects with psychiatric disorders (Fig. 2). Thus, atypical social behavior in canines can be considered as an "abnormal" social behavior from the view of wild animals. Investigation for the biological mechanisms of how atypical social behavior has been evolved in canines may yield a novel insight for understanding the biological mechanisms of psychiatric 
disorders with altered social function including schizophrenia. In relation to this issue, it is notable that brains of canines seem to have evolved into an atypical condition and became closer to those of humans, as evidenced by spontaneously occurring Alzheimer's disease-like alterations in canine brains, but has never been reported in wild animals (60).

From the evolutionary perspective, the most important aspect of psychiatric disorders is that the disorders may have emerged as environmental adaptation or by-products of such adaptation processes. In this regard, the Barker hypothesis is of particular interest (61). The Barker hypothesis suggests that prenatal environment causes developmental adaptation in fetus to prepare for the expected environment in postnatal life. For instance, insufficient nutrition during pregnancy lead to lower metabolism in fetus to maximize energy conservation for survival with a small amount of foods in the expected postnatal environment. However, when such offspring with lower metabolism is exposed to normal nutrition after birth, the outcome is exceeding energy conservation over life, resulting in such diseases as cancer, diabetes, obesity, and cardiovascular diseases in adulthood. Therefore, it is discrepancy between prenatal and postnatal environmental conditions, but not alterations associated with only prenatal or postnatal condition, that causes diseases. Although the impacts of either prenatal or postnatal environment alone on neurodevelopment were extensively investigated, the impacts of prenatal and postnatal environmental interaction has been yet barely investigated. However, for instance, there is one study by Leuba and Rabinowicz showing that offspring born from dams with diet restriction during pregnancy exhibits decreased dendritic spine density in cortical neurons when offspring was raised in normal postnatal nutrition. However, such offspring was grown with restricted 
nutrition, spine density was normalized (62). Epidemiological studies have unveiled that antenatal maternal exposure to adverse environment such as famine, stress, and infection during pregnancy increases the risk of psychiatric disorders such as schizophrenia (63), autism (64), and ADHD (65) in offspring. More recently, transgenerational inheritance of epigenetic changes (66-68) and altered behavior associated with specific environmental factors (69-72) have been reported. Such transgenerational inheritance of epigenetic changes associated with prenatal environmental condition and altered neurodevelopment may therefore be biological bases for emergence of psychiatric disorders through evolution in humans.

\section{Conclusion}

We suggest that the current translation research approach utilizing animal models for understanding the biological mechanisms of such psychiatric disorders as schizophrenia has limitations. Investigation with the evolutionary perspective may open a new venue as an alternate of the current research strategy. 


\section{References}

[1] F. Plum, Prospects for research on schizophrenia. 3. Neurophysiology. Neuropathological findings, Neurosciences Research Program bulletin 10 (1972), pp. 384-388.

[2] P.S. Wang, R. Heinssen, M. Oliveri, A. Wagner and W. Goodman, Bridging bench and practice: translational research for schizophrenia and other psychotic disorders, Neuropsychopharmacology 34 (2009), pp. 204-212.

[3] J.D. Cohen and T.R. Insel, Cognitive neuroscience and schizophrenia: translational research in need of a translator, Biol Psychiatry 64 (2008), pp. 2-3.

[4] Muly EC III, Szigeti K and Goldman-Rakic PS, D1 receptor in interneurons of macaque prefrontal cortex: distribution and subcellular localization., Journal of Neuroscience 18 (1998), pp. 10553-10565.

[5] E.J. Nestler and S.E. Hyman, Animal models of neuropsychiatric disorders, Nat Neurosci 13 (2010), pp. 1161-1169.

[6] B.K. Lipska and D.R. Weinberger, To model a psychiatric disorder in animals: schizophrenia as a reality test, Neuropsychopharmacology 23 (2000), pp. 223-239.

[7] H. Hafner, K. Maurer, W. Loffler and A. Riecher-Rossler, The influence of age and sex on the onset and early course of schizophrenia, $\mathrm{Br} J$ Psychiatry 162 (1993), pp. 80-86.

[8] B.K. Lipska, G.E. Jaskiw and D.R. Weinberger, Postpubertal emergence of hyperresponsiveness to stress and to amphetamine after neonatal excitotoxic hippocampal damage: a potential animal model of schizophrenia, 
Neuropsychopharmacology 9 (1993), pp. 67-75.

[9] M. Schneider and M. Koch, Behavioral and morphological alterations following neonatal excitotoxic lesions of the medial prefrontal cortex in rats, Exp Neurol 195 (2005), pp. 185-198.

[10] Saunders RC, Kolachana BS, Bachevalier J and Weinberger DR, Neonatal lesions of the medial temporal lobe disrupt prefrontal cortical regulation of striatal dopamine., Nature 393 (1998), pp. 169-171.

[11] H. Moore, J.D. Jentsch, M. Ghajarnia, M.A. Geyer and A.A. Grace, A neurobehavioral systems analysis of adult rats exposed to methylazoxymethanol acetate on E17: implications for the neuropathology of schizophrenia, Biol Psychiatry 60 (2006), pp. 253-264.

[12] L. Zuckerman, M. Rehavi, R. Nachman and I. Weiner, Immune activation during pregnancy in rats leads to a postpubertal emergence of disrupted latent inhibition, dopaminergic hyperfunction, and altered limbic morphology in the offspring: a novel neurodevelopmental model of schizophrenia, Neuropsychopharmacology 28 (2003), pp. 1778-1789.

[13] J.D. Gambill and C. Kornetsky, Effects of chronic d-amphetamine on social behavior of the rat: implications for an animal model of paranoid schizophrenia, Psychopharmacology (Berl) 50 (1976), pp. 215-223.

[14] D.C. Javitt and S.R. Zukin, Recent advances in the phencyclidine model of schizophrenia, Am J Psychiatry 148 (1991), pp. 1301-1308.

[15] M. Kvajo, H. McKellar and J.A. Gogos, Avoiding mouse traps in schizophrenia genetics: lessons and promises from current and emerging mouse models, Neuroscience 211 (2012), pp. 136-164. 
[16] L. Lu, T. Mamiya, T. Koseki, A. Mouri and T. Nabeshima, Genetic animal models of schizophrenia related with the hypothesis of abnormal neurodevelopment, Biological \& pharmaceutical bulletin 34 (2011), pp. 1358-1363.

[17] B. Moghaddam and B.W. Adams, Reversal of phencyclidine effects by a group II metabotropic glutamate receptor agonist in rats, Science 281 (1998), pp. 1349-1352.

[18] G. Tsai and J.T. Coyle, Glutamatergic mechanisms in schizophrenia, Annual review of pharmacology and toxicology 42 (2002), pp. 165-179.

[19] P.N. Vinson and P.J. Conn, Metabotropic glutamate receptors as therapeutic targets for schizophrenia, Neuropharmacology 62 (2012), pp. 1461-1472.

[20] S. Chaki, Group II metabotropic glutamate receptor agonists as a potential drug for schizophrenia, Eur J Pharmacol 639 (2010), pp. 59-66.

[21] S.T. Patil, L. Zhang, F. Martenyi, et al., Activation of mGlu2/3 receptors as a new approach to treat schizophrenia: a randomized Phase 2 clinical trial, Nat Med 13 (2007), pp. 1102-1107.

[22] G.N. Elston, R. Benavides-Piccione and J. DeFelipe, The pyramidal cell in cognition: a comparative study in human and monkey, J Neurosci 21 (2001), p. RC163.

[23] P.J. Harrison, The neuropathology of schizophrenia. A critical review of the data and their interpretation, Brain 122 ( Pt 4) (1999), pp. 593-624.

[24] B. Elvevag and T.E. Goldberg, Cognitive impairment in schizophrenia is the core of the disorder, Crit Rev Neurobiol 14 (2000), pp. 1-21.

[25] R. O'Carroll, Cognitive impairment in schizophrenia., Adv Psychiatric Treat 6 
(2000), pp. 161-168.

[26] D.H. Holstein, F.X. Vollenweider, M.A. Geyer, P.A. Csomor, N. Belser and D. Eich, Sensory and sensorimotor gating in adult attention-deficit/hyperactivity disorder (ADHD), Psychiatry Res 205 (2013), pp. 117-126.

[27] W. Perry, A. Minassian, B. Lopez, L. Maron and A. Lincoln, Sensorimotor gating deficits in adults with autism, Biol Psychiatry 61 (2007), pp. 482-486.

[28] S. Kohl, K. Heekeren, J. Klosterkotter and J. Kuhn, Prepulse inhibition in psychiatric disorders--apart from schizophrenia, Journal of psychiatric research 47 (2013), pp. 445-452.

[29] H.B. van der Worp, D.W. Howells, E.S. Sena, et al., Can animal models of disease reliably inform human studies?, PLoS medicine 7 (2010), p. e1000245.

[30] P.S. Jensen, D. Mrazek, P.K. Knapp, et al., Evolution and revolution in child psychiatry: ADHD as a disorder of adaptation, J Am Acad Child Adolesc Psychiatry 36 (1997), pp. 1672-1679; discussion 1679-1681.

[31] B. Crespi and C. Badcock, Psychosis and autism as diametrical disorders of the social brain, Behav Brain Sci 31 (2008), pp. 241-261; discussion 261-320.

[32] B. Crespi, P. Stead and M. Elliot, Evolution in health and medicine Sackler colloquium: Comparative genomics of autism and schizophrenia, Proc Natl Acad Sci U S A 107 Suppl 1 (2010), pp. 1736-1741.

[33] J. Wagemans, J.H. Elder, M. Kubovy, et al., A century of Gestalt psychology in visual perception: I. Perceptual grouping and figure-ground organization, Psychol Bull 138 (2012), pp. 1172-1217.

[34] G.V. Barrett, P.A. Cabe and C.L. Thornton, Visual functioning and embedded figures test performance, Perceptual and motor skills 26 (1968), p. 40. 
[35] J. Parnas, P. Vianin, D. Saebye, L. Jansson, A. Volmer-Larsen and P. Bovet, Visual binding abilities in the initial and advanced stages of schizophrenia, Acta Psychiatr Scand 103 (2001), pp. 171-180.

[36] M.A. Bellgrove, A. Vance and J.L. Bradshaw, Local-global processing in early-onset schizophrenia: evidence for an impairment in shifting the spatial scale of attention, Brain Cogn 51 (2003), pp. 48-65.

[37] F. Happe and U. Frith, The weak coherence account: detail-focused cognitive style in autism spectrum disorders, Journal of autism and developmental disorders 36 (2006), pp. 5-25.

[38] R.A. Almeida, J.E. Dickinson, M.T. Maybery, J.C. Badcock and D.R. Badcock, A new step towards understanding Embedded Figures Test performance in the autism spectrum: the radial frequency search task, Neuropsychologia 48 (2010), pp. 374-381.

[39] E. Courchesne and K. Pierce, Why the frontal cortex in autism might be talking only to itself: local over-connectivity but long-distance disconnection, Curr Opin Neurobiol 15 (2005), pp. 225-230.

[40] D. Neary, J. Snowden and D. Mann, Frontotemporal dementia, Lancet neurology 4 (2005), pp. 771-780.

[41] J.C. Mell, S.M. Howard and B.L. Miller, Art and the brain: the influence of frontotemporal dementia on an accomplished artist, Neurology 60 (2003), pp. 1707-1710.

[42] K.E. Stephan, K.J. Friston and C.D. Frith, Dysconnection in schizophrenia: from abnormal synaptic plasticity to failures of self-monitoring, Schizophr Bull 35 (2009), pp. 509-527. 
[43] A.S. Meyer-Lindenberg, R.K. Olsen, P.D. Kohn, et al., Regionally specific disturbance of dorsolateral prefrontal-hippocampal functional connectivity in schizophrenia, Arch Gen Psychiatry 62 (2005), pp. 379-386.

[44] G. Winterer, R. Coppola, M.F. Egan, T.E. Goldberg and D.R. Weinberger, Functional and effective frontotemporal connectivity and genetic risk for schizophrenia, Biol Psychiatry 54 (2003), pp. 1181-1192.

[45] T. Grandin and C. Johnson, Animals in translation, Harcourt, New York, NY (2005).

[46] L. Wing, The history of ideas on autism: legends, myths and reality, Autism 1 (1997), pp. 13-23.

[47] D.C. Penn and D.J. Povinelli, On the lack of evidence that non-human animals possess anything remotely resembling a 'theory of mind', Philos Trans $R$ Soc Lond B Biol Sci 362 (2007), pp. 731-744.

[48] U. Frith, Mind blindness and the brain in autism, Neuron 32 (2001), pp. 969-979.

[49] M. Brune, "Theory of mind" in schizophrenia: a review of the literature, Schizophr Bull 31 (2005), pp. 21-42.

[50] L. Harrington, R.J. Siegert and J. McClure, Theory of mind in schizophrenia: a critical review, Cognitive neuropsychiatry 10 (2005), pp. 249-286.

[51] E. Pousa, R. Duno, G. Brebion, A.S. David, A.I. Ruiz and J.E. Obiols, Theory of mind deficits in chronic schizophrenia: evidence for state dependence, Psychiatry Res 158 (2008), pp. 1-10.

[52] C.D. Frith and R. Corcoran, Exploring 'theory of mind' in people with schizophrenia, Psychol Med 26 (1996), pp. 521-530. 
[53] N. Tinbergen, On aims and methods of ethology, Zeitschrift fur Tierpsychologie 20 (1963), pp. 410-433.

[54] R.A. Power, S. Kyaga, R. Uher, et al., Fecundity of patients with schizophrenia, autism, bipolar disorder, depression, anorexia nervosa, or substance abuse vs their unaffected siblings, JAMA psychiatry 70 (2013), pp. 22-30.

[55] T.C. Kyziridis, Notes on the history of schizophrenia, German J Psychiatry 8 (2005), pp. 42-58.

[56] B. Crespi, K. Summers and S. Dorus, Adaptive evolution of genes underlying schizophrenia, Proceedings 274 (2007), pp. 2801-2810.

[57] A. Stevens and J. Price, Evolutionary psychiatry: A new beginning, Routledge, London, UK (2000).

[58] P. Pongracz, A. Miklosi, E. Kubinyi, K. Gurobi, J. Topal and V. Csanyi, Social learning in dogs: the effect of a human demonstrator on the performance of dogs in a detour task., Anim Behav 62 (2001), pp. 1109-1107.

[59] B. Hare, M. Brown, C. Williamson and M. Tomasello, The domestication of social cognition in dogs, Science 298 (2002), pp. 1634-1636.

[60] M.N. Bosch, M. Pugliese, J. Gimeno-Bayon, M.J. Rodriguez and N. Mahy, Dogs with cognitive dysfunction syndrome: a natural model of Alzheimer's disease, Current Alzheimer research 9 (2012), pp. 298-314.

[61] D.J. Barker, Maternal nutrition, fetal nutrition, and disease in later life, Nutrition (Burbank, Los Angeles County, Calif 13 (1997), pp. 807-813.

[62] G. Leuba and T. Rabinowicz, Long-term effects of postnatal undernutrition and maternal malnutrition on mouse cerebral cortex. II. Evolution of dendritic branchings and spines in the visual region, Exp Brain Res 37 (1979), pp. 
299-308.

[63] D.A. Lewis and P. Levitt, Schizophrenia as a disorder of neurodevelopment, Annu Rev Neurosci 25 (2002), pp. 409-432.

[64] H. Gardener, D. Spiegelman and S.L. Buka, Prenatal risk factors for autism: comprehensive meta-analysis, Br J Psychiatry 195 (2009), pp. 7-14.

[65] T.D. Banerjee, F. Middleton and S.V. Faraone, Environmental risk factors for attention-deficit hyperactivity disorder, Acta Paediatr 96 (2007), pp. 1269-1274.

[66] T.B. Franklin, H. Russig, I.C. Weiss, et al., Epigenetic transmission of the impact of early stress across generations, Biol Psychiatry 68 (2010), pp. 408-415.

[67] D.M. Dietz, Q. Laplant, E.L. Watts, et al., Paternal transmission of stress-induced pathologies, Biol Psychiatry 70 (2011), pp. 408-414.

[68] L. Daxinger and E. Whitelaw, Understanding transgenerational epigenetic inheritance via the gametes in mammals, Nature reviews 13 (2012), pp. 153-162.

[69] R.C. Painter, C. Osmond, P. Gluckman, M. Hanson, D.I. Phillips and T.J. Roseboom, Transgenerational effects of prenatal exposure to the Dutch famine on neonatal adiposity and health in later life, Bjog 115 (2008), pp. 1243-1249.

[70] C. Bertram, O. Khan, S. Ohri, D.I. Phillips, S.G. Matthews and M.A. Hanson, Transgenerational effects of prenatal nutrient restriction on cardiovascular and hypothalamic-pituitary-adrenal function, J Physiol 586 (2008), pp. 2217-2229.

[71] M.M. Brouwers, W.F. Feitz, L.A. Roelofs, L.A. Kiemeney, R.P. de Gier and N. Roeleveld, Hypospadias: a transgenerational effect of diethylstilbestrol?, Human reproduction (Oxford, England) 21 (2006), pp. 666-669.

[72] E. Zambrano, P.M. Martinez-Samayoa, C.J. Bautista, et al., Sex differences in 
transgenerational alterations of growth and metabolism in progeny (F2) of female offspring (F1) of rats fed a low protein diet during pregnancy and lactation, J Physiol 566 (2005), pp. 225-236. 


\section{Figure Legends}

Figure 1. A schematic diagram illustrating the process of translational research. Studies with schizophrenia patients identify potential genetic and molecular changes associated with the disorder. Based on these findings, manipulations are given in animals to examine how alterations cause alterations in animals to further understand underlying biological mechanisms. A new pharmacological or other therapeutic method is then developed.

Figure 2. A schematic diagram illustrating the relationship between normal subject and subjects with psychiatric disorders.

Figure 3. Examples of (a) gestalt perception (Kanizsa's triangle) and (b) embedded figures test. In figure (a), an illusionary triangle can be seen in the middle. In figure (b), the above figure is hidden in the bottom figure.

Figure 4. Schematic diagrams of altered neural network connections between the PFC and other brain areas in (a) autism and (b) schizophrenia. In autism, augmented over-connection (bold lines with arrows) of the local network within the PFC, whereas weakened connection (disconnectivity, dashed lines with arrows) between the PFC and other brain structures (denoted as A, B, C... in the figure) have been suggested. In contrast, in schizophrenia, altered connection (dysconnectivity) between the PFC and other brain structures such as abnormal presence of functional connectivity, which is absent in normal subjects, has been suggested. 
Figure 5. A schematic diagram illustrating how the current translational research with an animal model approach and our proposed research with a comparative approach are different. The current research strategy is to investigate the biological mechanisms underlying psychiatric disorders with presumption that fundamental aspects of brains of animals and humans are similar (the overlapping area in the figure). In contrast, an alternate approach is to explore how brains of animals and humans are different to understand psychiatric disorders as unique conditions occurring in humans. 
Studies with

schizophrenia patients

Genes A, B, C...

Molecular changes $a, b, c .$.

Development of pharmacological or other therapeutic treatments

\section{Behavioral changes}

Augmented response to amphetamine

Decreased interaction with mates

Prepulse inhibition deficit

Cognitive dysfunction

etc.

Molecular/anatomical/physiological changes

Decreased GABA neurons in the PFC

Decreased number of spines in PFC neurons

Increased dopamine release in the striatum etc.

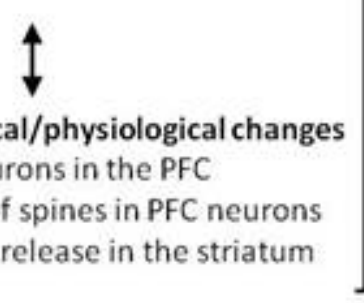

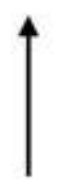

Underlying the mechanisms

$\longrightarrow$ underlying schizophrenia 
Subjects with psychiatric conditions

(minority of population)

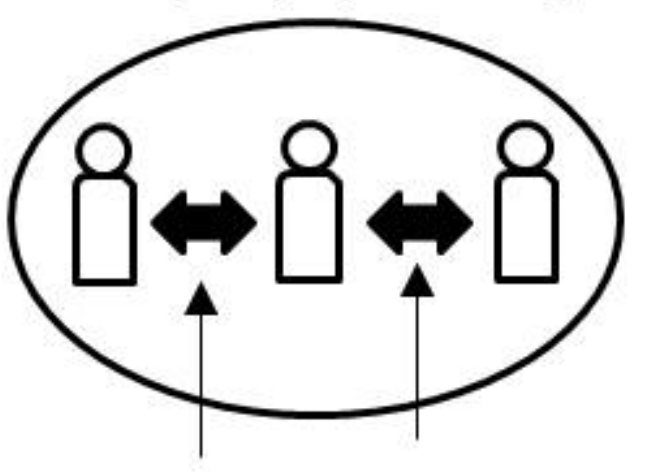

Normal relationship within the group

Behavior of nondiagnosed subjects also look abnormal Non-diagnosed subjects (Majority of population) from subjects with psychiatric conditions.

Psychiatric conditions look abnormal from nondiagnosed subjects.

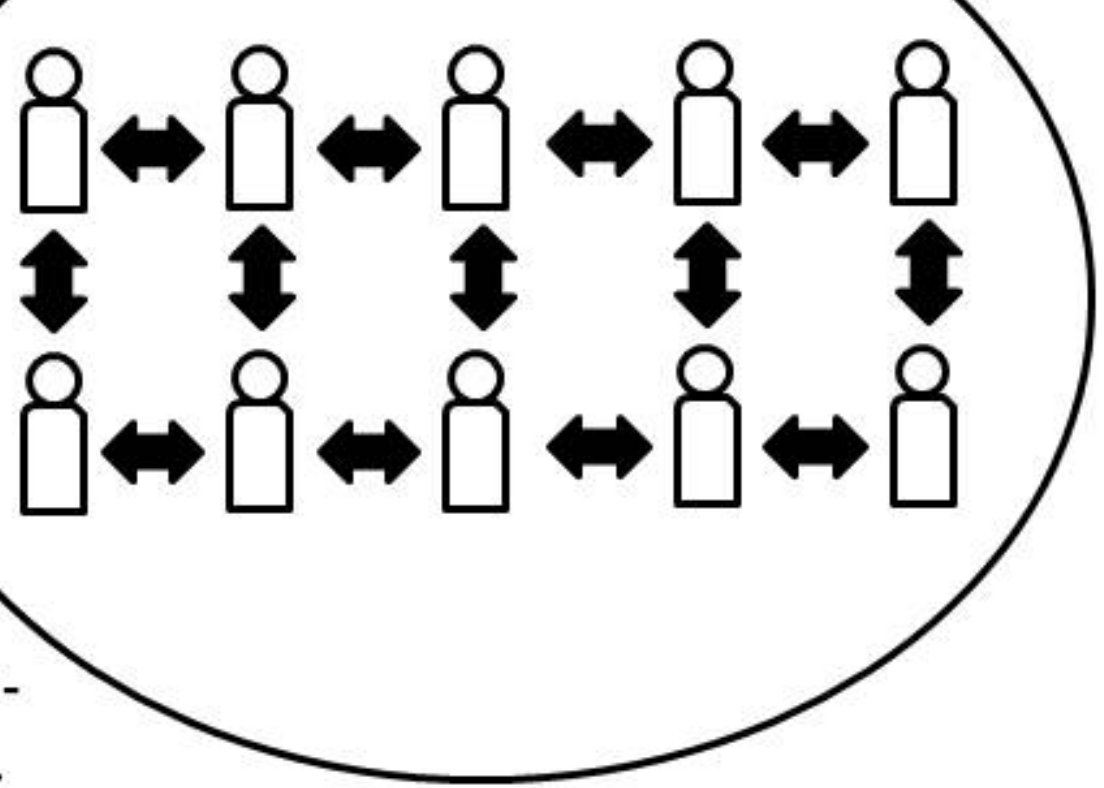

Normal relationship within the group 


\section{a}
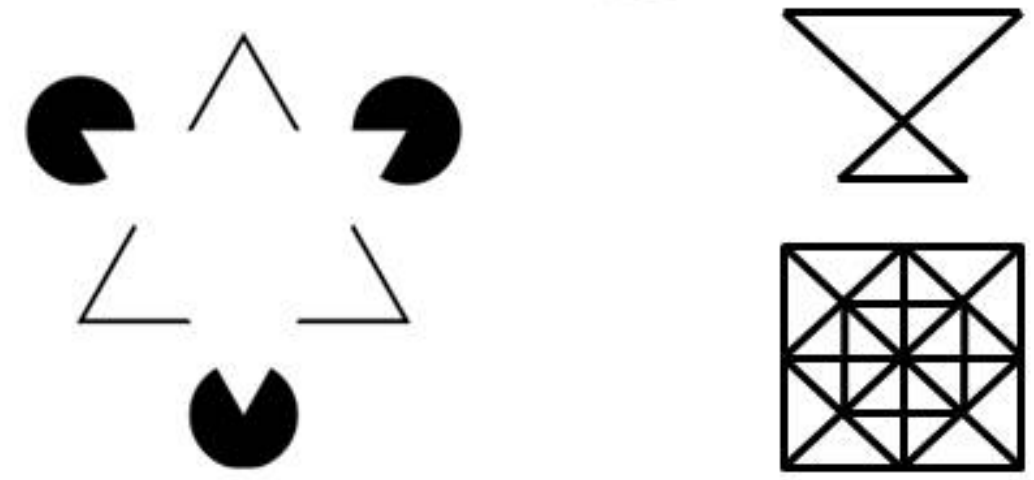

Figure 3 - Lee \& Goto 


\section{a}

\section{Autism}

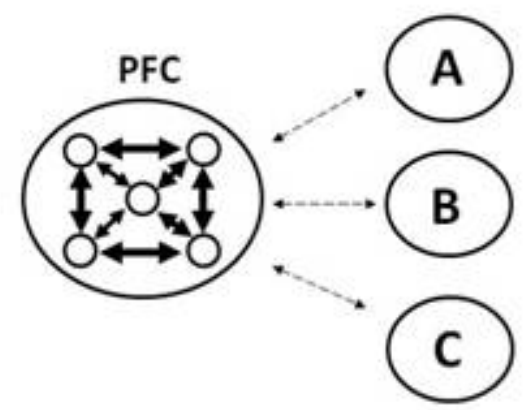

Disconnectivity
Schizophrenia

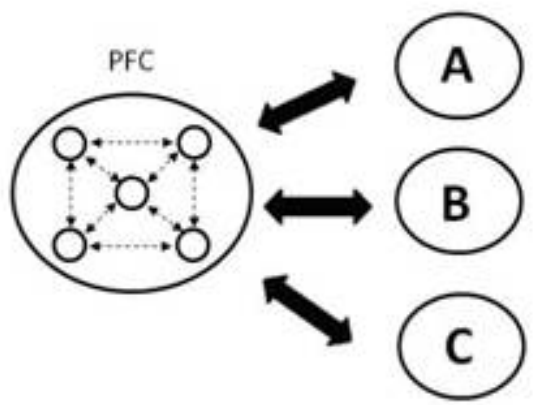

Dysconnectivity

Figure 4 - Lee \& Goto 


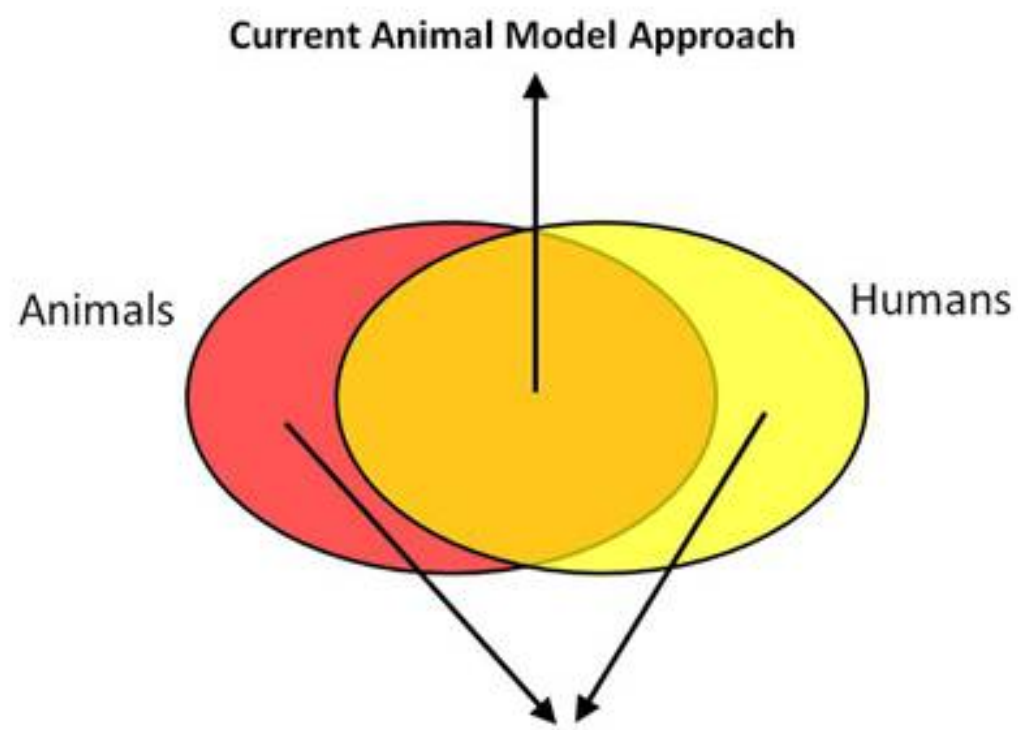

Areas that have been ignored 